\title{
A Load-Balancing Power Scheduling System for Renewables- Based Virtual Power Plant
}

\author{
Chennjung Huang ${ }^{1,2, a}$, Anfeng Liu, b, Kaiwen $\mathrm{Hu}^{2, ~ c}$, Juiting Hsiao ${ }^{1, \mathrm{~d}}$, \\ Liangchun Chen ${ }^{3,}$, , Chihting Chen ${ }^{1, f}$ \\ ${ }^{1}$ Department of Computer Science \& Information Engineering, National Dong Hwa University, \\ China. \\ ${ }^{2}$ Department of Electrical Engineering, National Dong Hwa University, China. \\ ${ }^{3}$ Department of Management, Fo Guang University, Yilan, Taiwan. \\ acjhuang@gms.ndhu.edu.tw, ban0922456111@gmail.com, cdrive55555@gmail.com, \\ dyji4j4@gmail.com, ef85170999999@gmail.com, fleaveall0207@gmail.com
}

\begin{abstract}
In the recent literature, the architecture of virtual power plant was proposed to replace the current smart grid in the future. However, the energy sharing concept and the uncertainties of intermittent energy sources will cause the short-term energy management for the virtual power plant much more complicated than the current centralized control energy management for traditional power generation system. We thus propose a hierarchical day-ahead power scheduling system based on the architecture of virtual power plant in this work to tackle the complex short-term energy management problems. A reallocation mechanism is presented to allocate excess electricity generated in a community virtual power plant to others facing with power supply shortage, and the maximal usage of renewables and reduction of the burden on community virtual power plants can be achieved accordingly. The experimental results show that the proposed work can mitigate the dependency on traditional power generation, and balance peak and off-peak period load of electricity market.
\end{abstract}

Keywords: virtual power plant, renewables, power scheduling, data mining, optimization.

\section{Introduction}

Similar to microgrids, virtual power plant (VPP) is a combination of distributed generators (DGs), controllable loads and energy storages [1]. The VPP aggregates the electrical output from a multitude of distributed energy resources and makes this supply available to the system operator. If requested, the VPP controls the immediate dispatch of the connected plants, thus contributing to grid reliability. The future VPP emphasizes the comprehensive utilization of energy in the whole power system by coordinating prosumers and minimizing the cost of the whole power system, but not only the energy of any single prosumer, which inevitably results in a certain amount of power exchange among the VPP members [2]. Therefore, how to accurately calculate the exchange power among prosumers in a distributed fashion is urgent for the energy management of the forthcoming VPP [3].

In the recent literature, the researches have already discussed VPPs and their challenges and opportunities in optimal scheduling issues in power markets. An approach that optimized their energy resources of a VPP in the day-ahead and the real-time energy markets was proposed in [1]. Trading strategies of a VPP in cooperation with its neighboring VPPs were addressed and a portfolio of interregional contracts was considered in [2] to maximize the energy trade opportunities of the VPP within a medium-term horizon. The resulting models were formulated as mixed-integer linear programming (MILP) problems that can be solved using off-the-shelf software packages. The feasibility of a VPP by means of local renewable energy power plant construction and the update of high efficiency appliances located in electricity customers was analyzed in [3]. The electricity market via changing feed-in tariffs and electricity price was built to analyze the economic performance of plant and demand sides. A bi-level scheduling model for VPPs with a large number of distributed thermostatically controlled loads and intermittent renewable energy is established in [4] to reduce the net exchange power deviation caused by the forecast error of renewable energy. Liu et. al combined interval and deterministic optimization together and adopted the combined approach to solve a VPP's 
dispatch problem [5]. The combined optimization not only maximized VPPs' deterministic profits under forecasted scenarios to estimate the VPP's most likely profits, but also maximized VPPs' profit intervals to manage uncertainties. A framework considering normal and contingency conditions for various VPPs was proposed in [6] to perform a day-ahead and intra-day generation scheduling by selecting the best DR programs. In this framework, the wind generations and the day-ahead and intraday electricity market prices were treated as the stochastic parameters. Since little research work presented short-term energy management systems that take load and renewables forecasts, V2G, battery energy storage system, and cooperation of multi-microgrids into consideration to realizing the benefits associated with future virtual power plant, a hierarchical day-ahead power scheduling system for renewables-based virtual power plant is thus proposed in this work to tackle the complex short term energy management problems. We first collect electricity consumption data from smart appliances used in households and predict power-generating capacity of renewable energy sources at the microgrid level. Then, the proposed power scheduling system is employed to schedule the usage of electricity for the customers by mainly considering the minimization of electricity cost of households and the efficiency of the use of distributed renewable energy. The remainder of this paper is organized as follows. Section 2 presents the proposed the day-ahead power scheduling system for proposed renewables-based virtual power plant. The simulation results are given in Section 3 . Conclusion is made in Section 4.

\section{The Day-Ahead Power Scheduling Algorithm}

A VPP framework with one main grid and several community virtual power plants (CVPPs) is considered in this work. The whole power system is first divided into different regions under the hierarchical framework. Each prosumer might have her/his own renewables, battery energy storage system and electric vehicles (EVs); and each region is managed by a CVPP, whereas the main grid at the top level is responsible for global scope. After the main grid initiates its day-ahead power scheduling main program, the main grid first requests the CVPPs to activate the first-round day-ahead power scheduling module. Each CVPP issues power scheduling requests to the prosumers downstream, and ask the prosumers to generate extra power as much as they can offer. The main grid then collects reported power data passed from the CVPPs downstream and compute the difference of power supply and demand in the whole power market. In case any power deficit appears at any time slot(s) day-ahead, the main grid will pass the power deficit data to the CVPPs to activate the second round of power scheduling.

During the second round of power scheduling of the CVPPs, the CVPPs request the prosumers downstream to save extra power at the battery of electrical vehicle or battery storage systems owned by the prosumers to make up for the gap between the power demand and power supply during the peak hours in the whole day-ahead electricity market. The prosumers who can offer extra power will give the electricity selling price to the CVPP upstream. After the main grid receives the list of dayahead hour-by-hour electricity selling price reported by the CVPPs, it will sort the electricity selling price list in the ascending order, and scheduling the power demand and supply to minimize the gap between the power demand and supply during peak hours.

After the main grid determines where to purchase the required electricity during peak hours based on the selling price set by the prosumers, the main grid will activate the third round of power scheduling. Accordingly, the prosumers who are chosen to sell the power to the main grid will activate the third round of power scheduling to save the extra power at the battery of electrical vehicle or battery storage systems as requested. Notably, the computational complexity for the traditional algorithm based on the central control increases dramatically if the coverage area of the power system is large. Thus, the proposed approach instead makes use of a hierarchical structure, so that the computational complexity can be reduced when compared with the traditional method. 


\section{Experimental Results and Analysis}

We ran a series of simulations by using python programming language to verify the feasibility and effectiveness of our proposed day-ahead renewables-based power scheduling system. We divide the whole power usage area into five regions, and each region had its own CVPP, which was governed by the main grid. Our load data, wind power and solar power data, and the data of EVs and battery storage systems were modified from real data to run the proposed scheduling program.

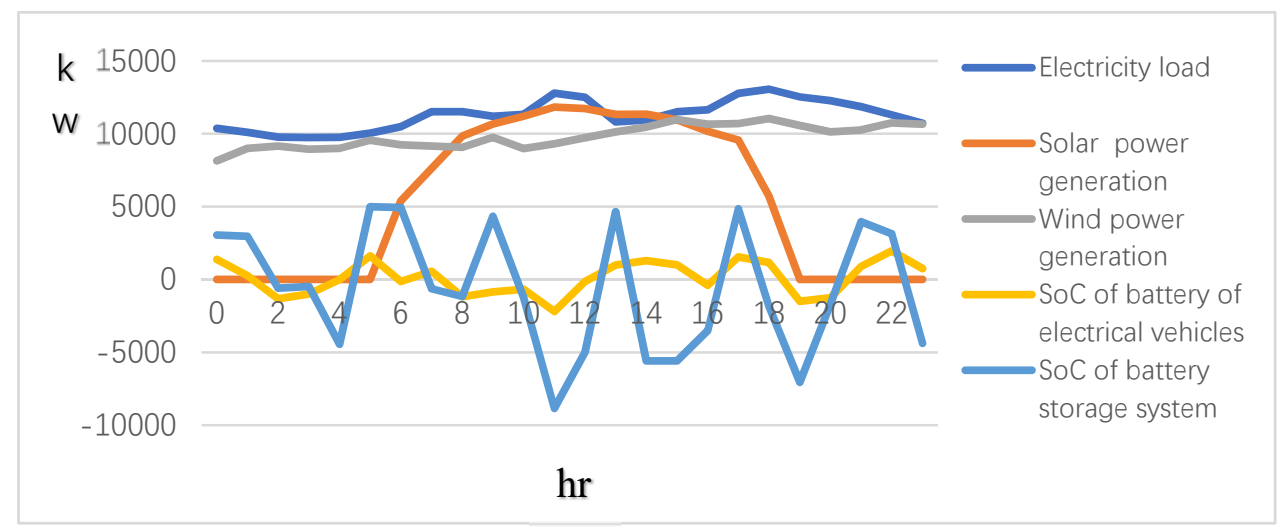

Fig. 1 Illustration of Day-Ahead Electricity Load, Renewables Generation, SoC of Battery of EVs, and SoC of Battery Storage System using Proposed Power Scheduling System

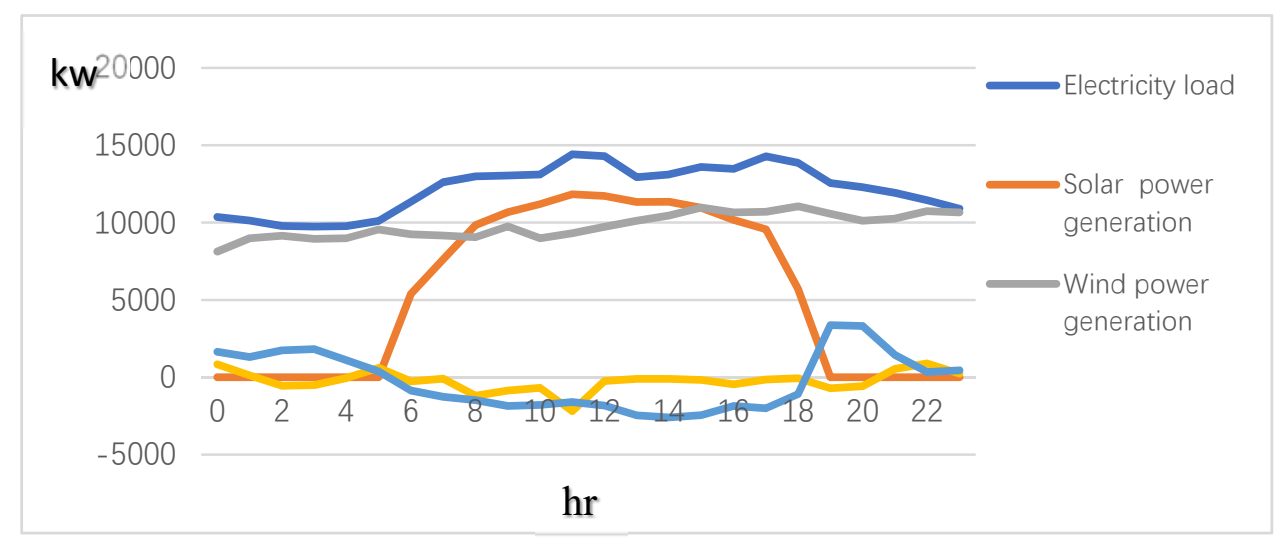

Fig. 2 Illustration of Electricity Load, Renewables Generation, SoC of Battery of EVs, and SoC of Battery Storage System without using Proposed Power Scheduling System

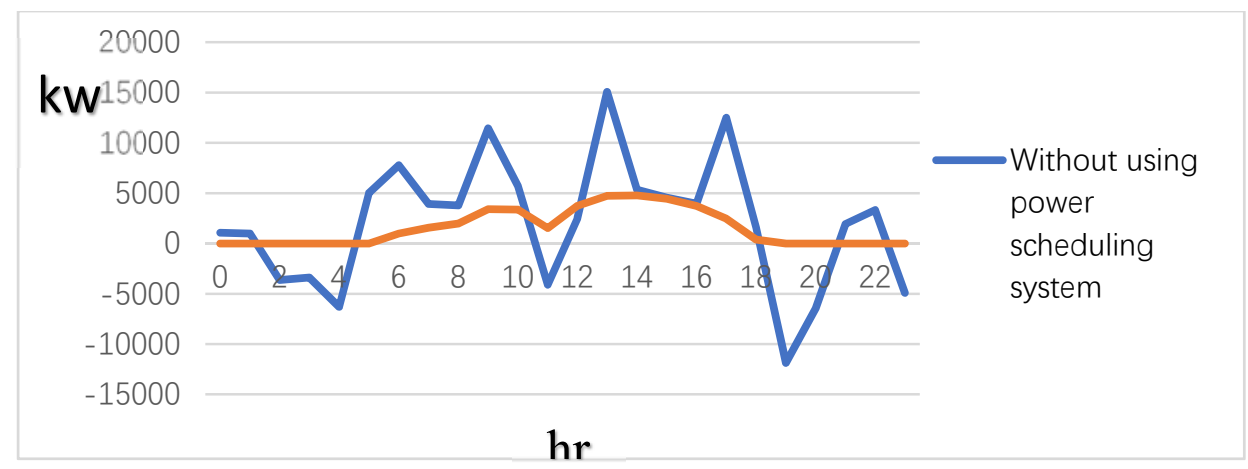

Fig. 3 Comparison of Power Surplus/Deficit Curve using and without using Proposed Power Scheduling System

Figs. 2 and 3 show the comparison of day-ahead electricity load, renewables generation, SoC of battery of electrical vehicles, and SoC of battery storage systems using and without using proposed power scheduling system, respectively. We can observe from Fig. 2 that similar peaks appear in the curve of electricity load and the aggregation of power generation and storage after using the proposed power scheduling system. Accordingly, these peaks assist in the balance on power supply and demand 
for the prosumers. On the contrary, the peaks in the curves of electricity load and renewables that appear at different time slot in Fig. 4 cause imbalance of on power supply and demand of the power system. The smoother shape of power surplus/deficit curve using the proposed power scheduling system as shown in Fig. 4 provides the evidence of the effectiveness of using proposed day-ahead power scheduling system.

\section{Conclusion}

In the recent literature, the architecture of virtual power plant was proposed to replace the current smart grid in the future. However, the energy sharing concept and the uncertainties of intermittent energy sources will cause the renewable-based short-term energy management for the future virtual power plant much more complicated than current centralized control short-term energy management for traditional power generation system. To tackle the forthcoming complicated short-term energy management problem of virtual power plant, a hierarchical day-ahead renewable-based power scheduling system is proposed in this work. Short-term electricity load and power generating capacity of renewables at the prosumer level is predicted first. Then, power scheduling at community virtual power plant level is employed to schedule the usage of electricity for the customers by reallocating excess electricity generated by some prosumers to others facing with power supply shortage. Accordingly, maximal usage of renewables and balancing the loading of prosumers during peak hours can be achieved. The experimental results reveal that the hierarchical day-ahead renewables-based power scheduling system proposed in this work can mitigate the dependency on traditional power plants effectively, and balance peak and off-peak period load of electricity market.

\section{Acknowledgements}

The authors would like to thank the National Science Council of the Republic of China, Taiwan for financially supporting this research under Contract Numbers MOST 106-2221-E-259-012 and MOST 107-2221-E-259-016.

\section{References}

[1]. Baringo, A., \& Baringo, L., A stochastic adaptive robust optimization approach for the offering strategy of a virtual power plant. IEEE Transactions on Power Systems, Vol. 32 (2017), No. 5, pp. 3492-3504.

[2]. Shabanzadeh, M., Sheikh-El-Eslami, M. K., \& Haghifam, M. R., An interactive cooperation model for neighboring virtual power plants. Applied energy, Vol. 200 (2017), pp. 273-289.

[3]. Li, Y., Gao, W., \& Ruan, Y., Feasibility of virtual power plants (VPPs) and its efficiency assessment through benefiting both the supply and demand sides in Chongming country, China. Sustainable Cities and Society, Vol. 35 (2017), pp. 544-551.

[4]. Wei, C., Xu, J., Liao, S., Sun, Y., Jiang, Y., Ke, D., \& Wang, J., A bi-level scheduling model for virtual power plants with aggregated thermostatically controlled loads and renewable energy. Applied Energy, Vol. 224 (2018), pp. 659-670.

[5]. Liu, Y., Li, M., Lian, H., Tang, X., Liu, C., \& Jiang, C., Optimal dispatch of virtual power plant using interval and deterministic combined optimization. International Journal of Electrical Power \& Energy Systems, Vol. 102 (2018), pp. 235-244.

[6]. Hooshmand, R. A., Nosratabadi, S. M., \& Gholipour, E., Event-based scheduling of industrial technical virtual power plant considering wind and market prices stochastic behaviors-A case study in Iran. Journal of Cleaner Production, Vol. 172 (2018), 1748-1764. 